\title{
Photodynamic and Anti- VEGF Therapy for Polypoidal Choroidal Vasculopathy - 'Real World' Outcomes in a Caucasian Population.
}

Kenneth M. Gilmour ( $\sim$ Kenneth.gilmour@ggc.scot.nhs.uk)

Gartnavel General Hospital

David Young

University of Strathclyde

Aaron Jamison

Gartnavel General Hospital

Monica Precup

Gartnavel General Hospital

David F. Gilmour

Gartnavel General Hospital

\section{Research Article}

Keywords: Anti-VEGF, Caucasian, Polypoidal choroidal vasculopathy, Photodynamic therapy, Real-world outcomes

Posted Date: July 7th, 2021

DOI: https://doi.org/10.21203/rs.3.rs-638281/v1

License: (1) This work is licensed under a Creative Commons Attribution 4.0 International License.

Read Full License 


\section{Abstract}

\section{Background}

The prevalence of polypoidal choroidal vasculopathy (PCV) is significantly higher amongst Asian populations compared to Caucasian, and evidence regarding the clinical outcomes of Caucasian patients is limited. This retrospective study sought to investigate real-world clinical outcomes of Caucasian PCV patients treated with polypoidal verteporfin photodynamic therapy (PDT) in combination with anti-VEGF therapy up to 36 months post treatment.

\section{Methods}

Consecutive PCV patients who received PDT between 2011 and 2017 were included. Mean change in visual acuity (VA) measured by ETDRS letter score and mean change in central subfield thickness (CST) were the main outcome measures. Data was collected at baseline, 3, 12, 24 and 36 months. Regression analyses were carried out on pre-treatment clinical features to determine if there were any factors associated with a good visual outcome (better than or equal to 70 ETDRS letters at 12 months).

Results

Seventy six patients (96\% Caucasian) were included in the analysis. Mean change in VA was $-1,-4$, and 0 ETDRS letters at 12,24 , and 36 months respectively. CST was reduced by a mean of $-51,-54$, and -55 microns at 12, 24, and 36 months. Better pre-treatment VA was the only pre-treatment clinical feature associated with a good visual outcome at 12 months (OR 1.16, $p<0.001$ ).

Conclusions

PDT in combination with anti-VEGF therapy maintains VA and may reduce anti-VEGF therapy burden in Caucasian patients with PCV. Better pre-treatment VA is associated with a good visual outcome.

\section{Background}

Polypoidal choroidal vasculopathy (PCV), also termed aneurysmal type 1 neovascularisation, ${ }^{1}$ is characterized by polyp-like aneurysmal dilations arising from branching vascular networks (BVN) underlying the retinal pigment epithelium (RPE).$^{2-4}$ Patients with PCV present with recurrent serosanguinous maculopathy of varying severity from massive haemorrhage to small retinal pigment epithelial detachments (PEDs) with a small amount of subretinal fluid (SRF). Characteristic optical coherence tomography (OCT) features include steep sided PEDs, irregular shallow PEDs (the double layer sign) and pachychoroid vessels. ${ }^{5}$ There is still controversy as to whether PCV is a sub-type of neovascular age related macular degeneration (nAMD), or whether it is a separate clinical entity. There are several demographic, clinical and angiographic features that support its classification as a distinct diagnosis. ${ }^{6,7} \mathrm{PCV}$ is not associated with typical AMD drusen, it is more prevalent amongst younger age 
groups and it is more prevalent amongst Asian and pigmented races. ${ }^{5,8,9}$ Aneurysmal dilatations can be seen in the early phases of indocyanine green angiography (ICGA) in cases of PCV and this remains the gold standard for making the diagnosis. ${ }^{5}$

Prevalence of PCV amongst Asian patients with presumed nAMD has been found to be as high as 22$54 \% .{ }^{10,11}$ In Caucasian populations this figure is smaller and studies have estimated prevalence between 4-22\%. ${ }^{8,11,12}$ Much of the literature investigating PCV outcomes stems from an Asian population base and less data exists assessing the outcomes of patients in Caucasian populations. Interestingly, a study of Caucasian nAMD patients who responded poorly to anti-VEGF treatment found the prevalence of PCV to be as high as $21.5 \% .^{13}$

PCV can be treated with anti-vascular endothelial growth factor (VEGF) monotherapy, with anti-VEGF and verteporfin photodynamic therapy (PDT) in combination, or PDT monotherapy. In terms of polyp closure, PDT has been shown to be superior to anti-VEGF, ${ }^{14}$ however it is thought constriction of choroidal vessels after PDT leads to hypoxia in both the choroid and retinal pigment epithelium which causes up-regulation of VEGF. ${ }^{15}$ Therefore giving concurrent anti-VEGF helps protect against the sequelae of choroidal and RPE ischaemia as well as treating the exudative changes resulting from neovascularization. ${ }^{16}$

Debate still remains as to which specific treatment regimen is the most effective. ${ }^{3}$ Studies have demonstrated the effectiveness of anti-VEGF monotherapy with ranibizumab, aflibercept and becavizumab as well as with PDT monotherapy. ${ }^{17-20}$ However, combination therapy is thought to have the highest success rate with regards to both polyp closure and improved visual acuity (VA). ${ }^{14,21}$ EVEREST $\|$ is the largest randomized controlled trial comparing combination therapy with anti-VEGF monotherapy. ${ }^{14}$ This trial demonstrated that PDT in combination with ranibizumab improved VA by 8.3 ETDRS letters after one year, compared to an improvement of 5.1 letters in the ranibizumab monotherapy group.

Retrospective studies on 'real world' patients can provide valuable data on the effectiveness of treatments in routine clinical practice. We report results from a study of 76 patients (78 eyes) treated for PCV with PDT +/- anti-VEGF therapy and followed up to 36 months post baseline PDT, $96 \%$ of whom were Caucasian. This is one of the largest studies reporting PCV outcomes in a predominately Caucasian population. The study investigates safety and efficacy outcomes for PDT in PCV, including a cohort of patients that received prior anti-VEGF treatment. In addition we investigated whether there were any underlying clinical features which were associated with a good VA outcome.

\section{Methods}

Consecutive patients who received PDT for a diagnosis of PCV in a tertiary referral centre between 2011 and 2017 were included in this retrospective study. The patients were either new referrals that were treatment naïve to both anti-VEGF and PDT or were refractory to anti-VEGF monotherapy for presumed nAMD, and were referred for investigation for PCV with ICGA, and subsequent rescue PDT. Patients were 
identified as having PCV by a consultant ophthalmologist with a specialist interest in medical retina based on clinical findings and imaging with ICGA and OCT. Standard practice in our centre is to carry out ICGA for the majority of patients with type 1 neovascularisation or with OCT signs suggestive of PCV.

Data was collected at baseline (clinic visit immediately prior to first PDT), and as close to 3, 12, 24 and 36 month intervals as possible. Data collected included age, sex, VA measured by ETDRS letter score, number of anti-VEGF treatments prior to first PDT, and number of retreatments with anti-VEGF and PDT. The majority of the VA measurements were recorded using ETDRS letter score, however a small number were recorded in Snellen VA. Snellen measurements were converted to ETDRS letter score using a recognized method. ${ }^{22}$ Central subfield thickness (CST) was recorded at each time point, measured from the central $1 \mathrm{~mm}$ subfield using spectral domain OCT on the TOPCON 3D OCT-2000 (Topcon, Tokyo, Japan). In addition, baseline ICGA and fundal features were recorded including formation of polyps (single, cluster or string), location of polyps (foveal [within 500 microns of the foveal centre], extrafoveal or peripapillary), number of polyp areas (one or multiple), presence of any haemorrhage or exudate, and presence of massive haemorrhage (four disc areas or more).

This study included patients who had undergone long durations of treatment with anti-VEGF for presumed nAMD prior to treatment with rescue PDT. To investigate whether including this patient cohort had an effect on the outcomes of the patient population as a whole, a further analysis was undertaken to compare outcomes of treatment naïve patients (those who had never received PDT or anti-VEGF prior to presentation) with "previously treated" patients (all patients with prior exposure to anti-VEGF).

All patients received PDT by infusion of verteporfin $(6 \mathrm{mg} / \mathrm{m} 2)$ followed 15 minutes later by administration of diode laser $(690 \mathrm{~nm})$ with $600 \mathrm{~mW} / \mathrm{cm} 2$ irradiance and duration of $83 \mathrm{~s}(50 \mathrm{~mJ} / \mathrm{cm} 2$ fluence) over the polyp and BVN area that was identified on ICGA. ${ }^{23}$ Our standard management is to combine PDT with an initial course of three monthly anti-VEGF injections (ranibizumab or aflibercept). PDT monotherapy was planned for a minority of patients with less aggressive lesions, and lesions away from the foveal centre. In cases of persistent exudative changes additional anti-VEGF was continued monthly on a PRN basis until exudative changes resolved. If the treating clinician suspected that the polyps were still open and active, repeat ICGA was carried out with subsequent further PDT if indicated. Polyp closure rate was not investigated because ICGA was only carried out if clinically indicated.

Univariate logistic regression was carried out on the above pre-treatment features to determine if there were any factors associated with a good or poor VA outcome at 12 months. For the purposes of this analysis 70 letters or better was categorized as a good outcome (group 1), and worse than 70 letters was categorized as a poor outcome (group 2). A multivariate logistic regression model was then used to determine which predictors were independently associated with a good outcome. All analyses were done using Minitab (version 18) at a 5\% significance level.

The research described adhered to the tenets of the declaration of Helsinki, and no ethical approval was required for this study. 


\section{Results}

Baseline demographics of the 76 patients included in the study are described in Table 1 . The majority were female (68\%) and of a Caucasian background (96\%). The mean age at presentation was 73 years with a range of $18-87$ years. With regard to the PCV lesion characteristics $69 \%$ of eyes showed a cluster of polyps, and in $65 \%$ of cases polyps were present in only one area. Polyps were located in the peripapillary and extrafoveal regions in approximately equal proportions at around $39 \%$, with $18 \%$ of cases having polyps within $\mathbf{5 0 0}$ microns of the foveal centre. Haemorrhage was present in half of the patient cohort, with massive haemorrhage present in $5 \%$. Two thirds of patients were found to have macular exudation. From the 78 eyes recorded at baseline, data was available for 71, 59 and 41 eyes at 12, 24 and 36 months following PDT treatment respectively. At 36 months follow up there was a loss to follow up of 37 patients (39 eyes) as a result of patient death $(n=5)$, transfer of care back to referring clinician $(n=7)$, treatment success and subsequent discharge $(n=8)$, treatment failure and subsequent discharge $(n=11)$ and unknown loss to follow up $(n=6)$.

Combination treatment with PDT and anti-VEGF was given to $89 \%$ of cases with the remaining patients receiving PDT only without any need for anti-VEGF therapy. Of those treated with anti-VEGF, $29 \%$ received initial treatment with ranibizumab and $70 \%$ received aflibercept. A small number of patients required repeat PDT (12 patients) and one patient received a third PDT treatment during the follow up period. Table 2 describes changes in VA and CST during follow-up. Patients maintained a stable VA with a mean of 56 letters observed at baseline and at three years follow up respectively. CST was reduced by a mean of -51 microns at 12 months and this reduction was maintained up to 36 months. A subgroup analysis comparing results by polyp location showed that patients with a peripapillary polyp location had a median improvement in VA from 63 to 70 ETDRS letters at 12 months. Those with an extrafoveal polyp location had no change in median VA at 12 months, and those with a foveal polyp location had a drop in median VA from 56 to 53 letters.

Of 78 eyes treated with PDT, 40 eyes had received prior treatment with anti-VEGF and 38 eyes were treatment naïve. Patients previously treated with anti-VEGF were seen in the clinic for a median of 1.72 years prior to receiving PDT and received an average of 4.96 injections in the 12 months prior to PDT, which reduced to an average of 2.60 injections in the 12 months following PDT, and 2.19 injections in the 12-24 month follow-up period. Treatment naïve patients received an average of 3.21 injections over the 12 months following PDT, but this included a standard loading dose of three anti-VEGF injections. A small number $(n=7)$ of treatment naïve patients with peripapillary or lower risk extrafoveal lesions on ICGA were treated with monotherapy and had no exposure to anti-VEGF at 12 months follow-up. A subanalysis was performed to investigate any differences between treatment naïve patients and patients previously treated with anti-VEGF (Tables 3 and 4). Patients in the treatment naïve group had a mean loss of 5 ETDRS letters at 24 months and an improvement of 2 ETDRS letters at 36 months, with those in the previously treated group having a mean loss of 4 ETDRS letters at 24 and 36 months. The median VA prePDT and 12 months post PDT was significantly higher in the treatment naïve group. 
Table 5 displays univariate analyses carried out on pre-treatment factors to determine if there were any clinical features associated with a good visual outcome (VA $\geq 70$ ETDRS letters) at 12 months post PDT. Better pre-treatment VA was associated with a good visual outcome with an odds ratio of $1.16(p<0.001)$ indicating that for each additional one unit increase in pre-treatment $V A$, the odds of a good visual outcome increased by a factor of 1.16 . The odds ratio for a favourable outcome with peripapillary lesions was 3.23 compared to foveal lesions, however this finding did not reach statistical significance. There was also an association between haemorrhage at initial presentation with a poorer visual outcome (OR $0.40, p=0.067)$, but this again was not statistically significant.

There were two episodes of vision threatening complications immediately following PDT. They were both retinal pigment epithelium tears and resulted in reduction in VA of 22 letters and 3 letters at three month follow up, and 29 letters and 35 letters at 12 month follow up respectively. One case of anti-VEGF related endophthalmitis was excluded from follow-up on the basis it was not secondary to PDT. One patient suffered acute back pain at the time of the verteporfin infusion.

\section{Discussion}

This is one of the largest retrospective studies of PCV patients within a Caucasian population. The inclusion of all patients regardless of prior treatment or comorbidity reflects a real world population and increases our understanding of what realistic outcomes are for PCV patients treated with a combination of PDT and anti-VEGF therapy. However the authors acknowledge that this methodology may also be deemed a weakness as the study population was varied and included both treatment naïve patients and patients previously treated with anti-VEGF therapy. A sub-analysis was carried out to look at the differences between these two groups. In addition to the recognized limitations of a retrospective, nonrandomized study, there were three different consultants managing the patients included in the study which introduces the possibility of variations in practice even with best efforts to standardize treatment regimens.

PDT is more effective at inducing polyp closure when compared to anti-VEGF monotherapy, ${ }^{14}$ which may result in a reduced requirement for ongoing anti-VEGF injections with resulting benefits to patients and health service providers. Patients previously treated with anti-VEGF were assessed on their need for further anti-VEGF following initial treatment with PDT in this study. We showed that patients who had previously received multiple anti-VEGF injections as monotherapy had a reduced requirement for antiVEGF during the 24 months following rescue PDT.

Due to the lack of availability of ICGA facilities in many of the referring hospitals, many previously treated patients in our cohort were initially not known to have underlying PCV disease and were treated as nAMD with anti-VEGF monotherapy. A proportion of PCV patients will respond to anti-VEGF monotherapy, so these patients were not referred for consideration for PDT and therefore not included in this study. If patients did not respond as expected to anti-VEGF in their local hospital they were referred to our centre for further investigation with ICGA and treatment with rescue PDT. Therefore this group of PCV patients 
were a more difficult to treat group with a resistance to anti-VEGF monotherapy. We found that previously treated patients had a significantly poorer VA when compared to treatment naïve patients prior to PDT treatment, and from the regression analysis we observed that patients starting with poorer vision were more likely to have a poorer outcome following PDT. ICGA remains the gold standard for diagnosing PCV and its lack of availability can lead to a delay in diagnosis and optimal treatment. A study of 202 nAMD patients with sub-optimal response to anti-VEGF by Hatz et al ${ }^{13}$ found a high prevalence of PCV (21.5\%) and that without ICGA facilities the diagnosis of PCV can be easily missed, resulting in a delay for optimal treatment. There needs to be greater awareness and appropriate investigation of PCV in Caucasian populations, and early referral to centres with ICGA and PDT services is likely to lead to improved clinical outcomes.

Data describing clinical outcomes in Caucasian populations receiving treatment for PCV is limited. Agorogiannis et al found a stable VA in 48 eyes treated with combination therapy, PDT monotherapy or anti-VEGF monotherapy in a 12 month retrospective study. ${ }^{6}$ Davis et al found a mean increase in 2.5 letters in a retrospective review of 27 patients treated with combination therapy and followed up for a mean of 15.8 months. ${ }^{26}$ On the other hand there is a large body of evidence investigating the outcomes of PCV patients in primarily Asian populations. The landmark prospective RCTs EVEREST II and PLANET found better visual outcomes than were observed in our study. EVEREST II described an increase of 8.3 ETDRS letters at 12 months follow up for combination therapy with ranibizumab. ${ }^{14,24}$ The PLANET study showed an increase of 10.7 letters at 12 months follow up for treatment with aflibercept and rescue PDT. ${ }^{24}$ Retrospective studies of Asian PCV populations also support favourable visual outcomes at 12 months and beyond with combination treatment. A large meta-analysis by Wang et al investigating outcomes pooled from 543 eyes in two RCTs and nine retrospective studies showed improved mean VA up to 24 months post combination treatment in patients predominately of Asian origin. ${ }^{25}$ On average our collective patient group had either the same or less than five letter loss at each time point up to 36 months. When the treatment naïve group was looked at separately in the sub-analysis there was an average loss of five letters of vision up to 24 months follow-up, then a mean gain of two letters at 36 months. The previously treated group recorded a mean loss of four letters at 24 and 36 months, and the median VA was significantly lower pre and post treatment when compared to the treatment naïve group. A retrospective real world study by Ratanasukon et al of Asian PCV patients treated with rescue PDT following initial anti-VEGF found an overall mean loss of 0.95 letters after four years follow-up. ${ }^{10}$ Our data suggests that Caucasian PCV patients also have poorer outcomes when previously treated with antiVEGF therapy. In addition our results for treatment naïve PCV patients were worse than published controlled trials and real world retrospective studies suggesting that Caucasian PCV patients do not respond as well to combination treatment when compared to Asian patients. However more data is needed to corroborate this hypothesis.

The literature published assessing PCV outcomes amongst Caucasian populations is limited, however from the evidence that is known, and the findings in this study, it appears that visual outcomes are less favourable when compared to Asian populations. This may be due to treatment centres in countries with 
a predominately Caucasian population underdiagnosing PCV, which then leads to a delay in referral on to a tertiary centre for appropriate management. There needs to be greater awareness of PCV as the possible underlying diagnosis in Caucasian patients with neovascular maculopathy. In addition further research is needed to inform clinicians what the optimal management plan should be in this group of patients.

\section{Conclusion}

PDT in combination with anti-VEGF therapy maintains VA and may reduce anti-VEGF therapy burden in Caucasian patients with PCV. Better pre-treatment VA is associated with a good visual outcome. Visual outcomes of Caucasian PCV patients treated with PDT in combination with anti-VEGF therapy are less favourable when compared to Asian populations.

\section{Abbreviations / Acronyms}

PCV - Polypoidal choroidal vasculopathy

PDT - Photodynamic therapy

nAMD - Neovascular age related macular degeneration

CRT - Central retinal thickness

ICGA - Indocyanine green angiography

VA - Visual Acuity

ETDRS - Early treatment diabetic retinopathy study

SRF - Subretinal fluid

BVN - Branching vascular network

OCT - Optical coherence tomography

Anti- VEGF - Anti - vascular endothelial growth factor

PED - Pigment epithelial detachment

\section{Declarations}

Ethical Approval:

No Ethical approval was required for this study 
Consent for publication:

Not applicable

Availability of data and materials:

The datasets used and analysed during the current study are available from the corresponding author on reasonable request

Competing interests:

DFG has been supported to attend educational meetings by Novartis (November 2017) and Bayer (January 2019). AJ has been supported to attend an educational courses by Bayer.

Financial Support / funding:

None

Authors contribution:

KMG: Designed, collected and analysed data. Principal author in drafting and revising manuscript.

DY: Interpretation of data and statistical analysis

AJ: $\quad$ Conception and acquisition of data and revision of manuscript

MP: $\quad$ Conception and acquisition of data

DFG: Conception, analysis and interpretation of data, substantive manuscript revision and editing

Acknowledgements

Not applicable

\section{References}

1. Li M, Dolz-Marco R, Messinger JD, Sloan KR, Ferrara D, Curcio CA et al. Clinicopathologic Correlation of Aneurysmal Type 1 Neovascularization in Age-Related Macular Degeneration. Ophthalmol Retina. 2019;3(2):99-111.

2. Seong S, Choo HG, Kim YJ, Kim JY, Lee JH, Oh HS et al. Novel Findings of Polypoidal Choroidal Vasculopathy via Optical Coherence Tomography Angiography. Korean J Ophthalmology. 2019;33(1):54.

3. Ho C, Lai T. Current management strategy of polypoidal choroidal vasculopathy. Indian J Ophthalmol. 2018;66(12):1727. 
4. Li M, Huisingh C, Messinger J, Dolz-Marco R, Ferrara D, Freund KB et al. Histology of geographic atrophy secondary to age-related macular degeneration: a multilayer approach. Retina. 2018;38(10):1937-1953.

5. Cheung CMG, Lai TYY, Ruamviboonsuk P, Chen SJ, Chen Y, Freund KB et al. Polypoidal Choroidal Vasculopathy: Definition, Pathogenesis, Diagnosis, and Management. Ophthalmology. 2018;125(5):708-724.

6. Agorogiannis El, Pearce IA, Yadav S, Parry DG, Beare NAV. Clinical outcomes in Caucasian patients with polypoidal choroidal vasculopathy. Eye. 2018;32(11):1731.

7. Yannuzzi LA, Wong DW, Sforzolini BS, Goldbaum M, Tang KC, Spaide RF et al. Polypoidal choroidal vasculopathy and neovascularized age-related macular degeneration. Arch Ophthalmol. 1999;117(11):1503-1510.

8. Yadav S, Parry DG, Beare NAV, Pearce IA. Polypoidal choroidal vasculopathy: a common type of neovascular age-related macular degeneration in Caucasians. Br J Ophthalmol. 2017;101(10):1377.

9. Coppens G, Spielberg L, Leys A. Polypoidal choroidal vasculopathy, diagnosis and management. Bull Soc Belge Ophthalmol. 2011(317):39-44.

10. Ratanasukon M, Bhurayanontachai P, Jirarattanasopa P. Polypoidal choroidal vasculopathy (PCV): the 4-year review of the real-life treatment experiences. Clin Ophthalmol. 2018;12:2177-2181.

11. Bhoomibunchoo $C$, Yospaiboon $Y$, Thoongsuwan S, Rojanaporn D, Watanachai N, Jirarattanasopa $P$ et al. Idiopathic polypoidal choroidal vasculopathy in Thai patients with clinical and angiographic choroidal neovascularization. Clin Ophthalmol. 2017;11:317-322.

12. Lorentzen DT, Subhi LY, Sørensen LT. Prevalence of polypoidal choroidal vasculopathy in white patients with exudative age-related macular degeneration: systematic review and meta-analysis. Retina. 2018;38(12):2363-2371.

13. Hatz K, Prünte C. Polypoidal choroidal vasculopathy in Caucasian patients with presumed neovascular age-related macular degeneration and poor ranibizumab response. $\mathrm{Br} \mathrm{J}$ Ophthalmol. 2014;98(2):188.

14. Koh A, Lai TYY, Takahashi K. Efficacy and Safety of Ranibizumab With or Without Verteporfin Photodynamic Therapy for Polypoidal Choroidal Vasculopathy. A Randomized Clinical Trial.(Report). JAMA Ophthalmol. 2017;135(11):1206.

15. Tatar O, Adam A, Shinoda K, Stalmans P, Eckardt C, Lüke M et al. Expression of VEGF and PEDF in Choroidal Neovascular Membranes Following Verteporfin Photodynamic Therapy. Am J Ophthalmol. 2006;142(1):95-104.e1.

16. Sakurada Y, Sugiyama A, Tanabe N, Kikushima W, Kume A, lijima H. Choroidal thickness as a prognostic factor of photodynamic therapy with Aflibercept or Ranibizumab for polypoidal choroidal vasculopathy. Retina. 2017;37(10):1866-1872.

17. Oishi A, Miyamoto N, Mandai M, Honda S, Matsuoka T, Oh H. LAPTOP study: a 24-month trial of verteporfin versus ranibizumab for polypoidal choroidal vasculopathy. Ophthalmology. 2014 May;121(5):1151-2. 
18. Hikichi T. Six-year outcomes of antivascular endothelial growth factor monotherapy for polypoidal choroidal vasculopathy. Br J Ophthalmol. 2018;102(1):97.

19. Takayama K, Kaneko H, Kataoka K, Hattori K, Ra E, Tsunekawa T et al. Comparison between 1-year outcomes of aflibercept with and without photodynamic therapy for polypoidal choroidal vasculopathy: Retrospective observation study. PLoS ONE. 2017;12(5).

20. Yamashita A, Shiraga F, Shiragami C, Shirakata Y, Fujiwara A. Two-Year Results of Reduced-Fluence Photodynamic Therapy for Polypoidal Choroidal Vasculopathy. Am J Ophthalmol. 2013;155(1):96102.e1.

21. Wong CW, Cheung CMG, Mathur R, Li X, Chan CM, Yeo I, Wong E et al. Three-year results of polypoidal choroidal vasculopathy treated with photodynamic therapy: retrospective study and Systematic review. Retina. 2015;35(8):1577-1593.

22. Gregori Z, N., Feuer J, W., Rosenfeld J, P. Novel method for analyzing snellen visual acuity measurement. Retina. 2010;30(7):1046-1050.

23. Jamison A. Cauchi P. Gilmour DF. Photodynamic Therapy for Circumscribed Choroidal Haemangioma in a Scottish Cohort 2018;4:322-330. Ocul Oncol Pathol. 2018 2018;4:322-330.

24. Lee WK, lida T, Ogura Y, Chen SJ, Wong TY, Mitchell P et al. Efficacy and Safety of Intravitreal Aflibercept for Polypoidal Choroidal Vasculopathy in the PLANET Study: A Randomized Clinical Trial. JAMA Ophthalmol. 2018;136(7):786.

25. Wang W, He M, Zhang X. Combined Intravitreal Anti-VEGF and Photodynamic Therapy versus Photodynamic Monotherapy for Polypoidal Choroidal Vasculopathy: A Systematic Review and MetaAnalysis of Comparative Studies.(Research Article). PLoS ONE. 2014;9(10).

26. Davis SJ, Lauer A, Flaxel CJ. Polypoidal choroidal vasculopathy in white patients. Retina-J Ret Vit Dis. 2014;34(11):2185-2191.

\section{Tables}

Table 1. Baseline Demographics and Clinical Features $(n=78)$ 


\begin{tabular}{lll} 
Mean age at presentation (SD), years & 73 $(11)$ & \\
\hline Sex & Male & $31.6 \%$ \\
\hline & Female & $68.4 \%$ \\
\hline Ethnicity & Caucasian & $96.1 \%$ \\
\hline & Asian & $1.3 \%$ \\
\hline & African/Caribbean & $1.3 \%$ \\
\hline Polyp location & Unknown & $1.3 \%$ \\
\hline & Foveal & $17.9 \%$ \\
\hline Extrafoveal & $39.7 \%$ \\
\hline Polyp formation & Peripapillary & $38.4 \%$ \\
\hline & Unknown & $3.8 \%$ \\
\hline Cluster & $69.2 \%$ \\
\hline Macular exudate & String & $12.8 \%$ \\
\hline Single & $12.8 \%$ \\
\hline No. of polyp areas & Unknown & $5.1 \%$ \\
\hline & One & $65.4 \%$ \\
\hline & Multiple & $29.5 \%$ \\
\hline & Unknown & $5.1 \%$ \\
\hline & Yes & $66.7 \%$ \\
\hline & Unknown & $2.6 \%$ \\
\hline & Unknos & $2.6 \%$ \\
\hline & & $5.2 \%$ \\
\hline & & $2.6 \%$ \\
\hline
\end{tabular}

Table 2. Visual acuity and central retinal thickness at 0 to 36 months follow up. 


\begin{tabular}{llllll} 
Time from follow up (n) & $\begin{array}{l}\text { Baseline } \\
(78)\end{array}$ & $\begin{array}{l}3 \text { months } \\
(76)\end{array}$ & $\begin{array}{l}12 \text { months } \\
(71)\end{array}$ & $\begin{array}{l}24 \text { months } \\
(59)\end{array}$ & $\begin{array}{l}36 \text { months } \\
(41)\end{array}$ \\
\hline Median VA (range), letters & $60(0-85)$ & $60(4-87)$ & $59(0-90)$ & $60(0-89)$ & $60(0-85)$ \\
\hline Mean VA (SD), letters & $56(18)$ & $56(20)$ & $55(24)$ & $52(27)$ & $56(25)$ \\
\hline $\begin{array}{l}\text { Mean VA change from } \\
\text { baseline, letters }\end{array}$ & - & 0 & -1 & -4 & 0 \\
\hline $\begin{array}{l}\text { Median CST (range), microns } \\
\text { Mean CST (SD), microns }\end{array}$ & $\begin{array}{l}279(118- \\
604)\end{array}$ & $\begin{array}{l}218(145- \\
597)\end{array}$ & $\begin{array}{l}223(117- \\
665)\end{array}$ & $\begin{array}{l}222(132- \\
413)\end{array}$ & $222(121-$ \\
\hline $\begin{array}{l}\text { Mean CST change from } \\
\text { baseline, microns }\end{array}$ & - & -68 & -51 & -54 & -55
\end{tabular}

Table 3. Visual acuity and central retinal thickness at 0 to 36 months follow up in treatment naïve and previously treated subgroups.

\begin{tabular}{|c|c|c|c|c|c|}
\hline Time from follow up & Baseline & $\begin{array}{l}3 \\
\text { months }\end{array}$ & $\begin{array}{l}12 \\
\text { months }\end{array}$ & $\begin{array}{l}24 \\
\text { months }\end{array}$ & $\begin{array}{l}36 \\
\text { months }\end{array}$ \\
\hline Treatment Naïve subgroup & $\mathrm{n}=38$ & $n=37$ & $n=36$ & $\mathrm{n}=31$ & $\mathrm{n}=21$ \\
\hline Median VA (range), letters & $\begin{array}{l}63(24- \\
81)\end{array}$ & $65(4-87)$ & $69(5-85)$ & $61(0-88)$ & $\begin{array}{l}68(30- \\
85)\end{array}$ \\
\hline Mean VA (SD), letters & $62(16)$ & $61(19)$ & $61(23)$ & $57(26)$ & $64(16)$ \\
\hline $\begin{array}{l}\text { Mean VA change from baseline, } \\
\text { letters }\end{array}$ & - & -1 & -1 & -5 & +2 \\
\hline Previously treated subgroup & $\mathrm{n}=40$ & $n=39$ & $n=35$ & $n=28$ & $\mathrm{n}=20$ \\
\hline Median VA (range), letters & 54 & $\begin{array}{l}53(10- \\
83)\end{array}$ & $52(0-84)$ & $54(0-85)$ & $57(0-81)$ \\
\hline Mean VA (SD), letters & $51(19$ & $51(20)$ & $49(24)$ & $47(28)$ & $47(30)$ \\
\hline $\begin{array}{l}\text { Mean VA change from baseline, } \\
\text { letters }\end{array}$ & - & 0 & -2 & -4 & -4 \\
\hline
\end{tabular}

Table 4. Comparison of treatment naïve patients vs. patients previously treated with anti-VEGF at 12 months 


\begin{tabular}{|lllll|}
\hline & $\begin{array}{l}\text { Treatment Naive } \\
(\mathrm{n}=38) \\
\text { Median (IQR) }\end{array}$ & $\begin{array}{l}\text { Prior Treatment } \\
(\mathrm{n}=40) \\
\text { Median (IQR) }\end{array}$ & $95 \% \mathrm{Cl}$ & $\begin{array}{l}\mathrm{p}- \\
\text { value }\end{array}$ \\
\hline Age & $78(66,82)$ & $74(67,81)$ & $-2.6,6.5$ & 0.433 \\
\hline ETDRS Letter Score pre-PDT & $63(52,73)$ & $54(36,85)$ & $3.0,19.0$ & 0.008 \\
\hline CST pre-PDT & $277(222,327)$ & $288(232,355)$ & -53.0, & 0.532 \\
$\begin{array}{l}\text { ETDRS Letter Score 12 months } \\
\text { post-PDT }\end{array}$ & $69(51,79)$ & $52(35,73)$ & $3.0,25.0$ & 0.022 \\
\hline $\begin{array}{l}\text { CST 12 months post-PDT } \\
\text { (2) }\end{array}$ & $227(205,268)$ & $216(190,285)$ & -28.0, & 0.778 \\
\hline
\end{tabular}

Table 5. Univariate analyses to determine which pre-treatment factors were associated with a good visual outcome (VA $\geq 70$ Letters) at 12 months post PDT

\begin{tabular}{|c|c|c|c|c|c|}
\hline Clinical Characteristic & Variable 1 & Variable 2 & $\begin{array}{l}\text { Odds } \\
\text { Ratio }\end{array}$ & $\begin{array}{l}\text { Confidence } \\
\text { Interval }\end{array}$ & $\begin{array}{l}\mathrm{P} \\
\text { Value }\end{array}$ \\
\hline Age & - & - & 0.97 & $(0.93,1.01)$ & 0.140 \\
\hline Sex & Male & Female & 0.55 & $(0.18,1.66)$ & 0.281 \\
\hline $\begin{array}{l}\text { ETDRS Letter Score pre- } \\
\text { PDT }\end{array}$ & - & - & 1.16 & $(1.08,1.24)$ & $<0.001$ \\
\hline CST pre-PDT & - & - & 1.00 & $(0.99,1.00)$ & 0.921 \\
\hline $\begin{array}{l}\text { Total no. of Anti-VEGF pre- } \\
\text { PDT }\end{array}$ & - & - & 0.93 & $(0.84,1.02)$ & 0.106 \\
\hline Presence of Haemorrhage & Yes & No & 0.40 & $(0.15,1.08)$ & 0.067 \\
\hline Presence of Exudate & Yes & No & 1.28 & $(0.43,3.76)$ & 0.652 \\
\hline No. of Polyp areas & Single & Multiple & 2.10 & $(0.62,6.37)$ & 0.180 \\
\hline \multirow[t]{3}{*}{ Location of Polyp } & Foveal & Extrafoveal & 0.63 & $(0.14,2.88)$ & 0.211 \\
\hline & Peripapillary & Extrafoveal & 2.05 & $(0.70,5.97)$ & \\
\hline & Peripapillary & Foveal & 3.23 & $(0.71,14.61)$ & \\
\hline \multirow[t]{3}{*}{ Formation of Polyp } & Single & Cluster & 0.37 & $(0.07,1.95)$ & 0.459 \\
\hline & String & Cluster & 0.86 & $(0.21,3.43)$ & \\
\hline & String & Single & 2.33 & $(0.31,17.54)$ & \\
\hline
\end{tabular}

